Bolm Inst. oceanogr., S Paulo, 30(1):57-64, 1981

\title{
ANÄLISE ECONÔMICA DA PRODUÇÃO DA SARDINHA NA REGIÃO SUDESTE DO BRASIL
}

\author{
Yas unobu MATSUURA \\ Instituto Oceanogräfico da Universidade de São Paulo
}

\begin{abstract}
Synops is
A surplus-yield model of Schaefer $(1954$; 1957) was used to study a maximum sustainable yield of the Brazilian sardine. Applying the prices of sardine and operation cost, an economical yield of sardine was discussed. The maximum sustainable yield of sardine was estimated on 173 thousands metric tons gnd the optimum fishing effort was 28,292 sets of net. With the operation cost" of Cr\$ 71,040.00 per cruise, the price of sardine has to be higher than Cr\$10,00/kg.
\end{abstract}

\section{Int rodução}

A sardinha-verdadeira (Sardinella brasiliensis) è uma das espécies mais importantes na Região Sudeste do Brasil e está sendo explorada pela frota de traineiras, que conta com cerca de 300 embarcações nessa região. A exploração da sardinha foi iniciada em grande escala no começo da década de 60 , sendo que a maior produção foi atingida em 1973, num total de $228 \mathrm{mil}$ toneladas. Nos ûltimos três anos, a produção estabilizou-se ao nível de 140 a 150 mil toneladas ao ano.

Um dos objetivos do estudo da dinâmica de população é avaliar a potencialidade de recursos pesqueiros, a fim de se explorar racionalmente a máxima produtividade da região. Dispondo-se de dados estatísticos e biológicos, podemos estimar a produção máxima sustentável do estoque, através de vários modelos matemáticos. Para se evitar a sobrepesca e aproveitar racionalmente os recursos pesqueiros que o mar nos fornece, temos que administrar cientificamente as atividades pesqueiras. Por isso, as medidas de controle de pesca devem ser adotadas de acordo com os resultados da análise da população de peixes.

A pesca é uma atividade econômica que, através da captura de peixes, procura obter a maior rentabilidade, de modo que, para se estabelecer uma administração científica, não podemos ignorar o fator econômico. Neste sentido, foi feito um estudo da análise econômica da

Publ. no 521 do Inst. oceanogr. da Usp. produção da sardinha, baseado no modelo descritivo de Schaefer (1954; 1957), modificado por Shindo \& Yagi (1970). Isto foi possíve1, graças à disponibilidade de dados estatísticos e econômicos da sardinha, que vêm sendo acumulados, nos últimos anos, pelo Grupo Permanente de Estudo da Sardinha (PDP/SUDEPE).

Metodologia

Base teörica

0 modelo matemático para a avaliação do estado da população consta de dois métodos básicos: o modelo descritivo de Schaefer (1954; 1957) e o modelo analítico de Beverton \& Holt (1957). Este ültimo, é baseado na idéia formulada por Russel (1931). A dinâmica de população de peixes pode ser representada pelos quatro parâmetros básicos: recrutamento $(R)$, crescimento $(G)$, mortalidade natural (M), e mortalidade de pesca(F). A diferença entre o tamanho da população no início do ano $\left(\mathrm{P}_{0}\right)$ e o final do ano $\left(\mathrm{\sim}_{1}\right)$ é apresentada pela seguinte equação:

$$
P_{1}-P_{0}=(R+G)-(M+F)
$$

No modelo analítico, estudam-se os quatro elementos separadamente e, por isso, necessita-se de grande quantidade de informações sobre os parâmetros da população de peixes. 0 modelo descritivo, entretanto, baseia-se na ideia de que o incremento natural da população de peixes ( $i \cdot \bar{e} ., N=R+G-M)$ é determi- 
nado pelo tamanho da população; ou seja, a maior produtividade ocorre quando o tamanho da população diminue até um certo níve1. Diminuindo o tamanho da população com o aumento de captura, a população de peixes procura recuperar aquela parte perdida pela pesca. Por isso, controlando a intensidade de pesca

(= mortalidade de pesca, F), podemos obter um ponto de maior produtividade da população.

Para a aplicação do modelo descritivo, necessitamos somente dos dados de captura e esforço de pesca durante a1guns anos (pelo menos mais de 5 anos de observação). Pela simplicidade das informaçoes necessárias, o modelo descritivo è aplicado a vārias populações de peixes e normalmnete fornece valiosas informações sobre o estado da populacão.

\section{Descrição do modelo}

Analisando os dados de captura de atumlage no Oceano Pacífico leste, Schaefer (1957) observou que a captura por unidade de esforço de pesca (CPUE) diminuiu com o aumento do esforço de pesca durante o período de 1934 a 1955. Esta relação foi apresentada pela equação:

$$
\overline{\mathrm{U}}=\mathrm{a}-\mathrm{b} \mathrm{x}_{\mathrm{i}} \ldots \ldots \ldots \ldots \ldots \ldots
$$

onde $a$ e $b$ são constantes, $\overline{\mathrm{U}} \overline{\mathrm{e}}$ a captura por unidade de esforço de pesca e $x_{i}$ o esforço de pesca no ano $i$. A captura por unidade de esforço de pesca foi calculada com a equação:

$$
\overline{\mathrm{U}}=\mathrm{Y}_{i} / \mathrm{X}_{i}
$$

onde $\mathrm{Y}_{i}$ é a captura total no ano $i$. Juntândo-se as equações (1) e (2), obtemos a seguinte equação:

$$
\mathrm{Y}_{\mathrm{e}}=(\mathrm{a}-\mathrm{bX}) \mathrm{X}=\mathrm{aX}-\mathrm{bX}^{2} \ldots
$$

A relação entre captura $\left(Y_{e}\right)$ e esforço de pesca (X) é representada pela parábola da equação (3). 0 ponto máximo da parábola é chamado de ponto de equilíbrio $\left(\mathrm{P}_{\mathrm{e}}\right)$. A partir deste ponto, - aumento do esforço de pesca não ocasionaria um aumento de captura, mas sim sua diminuição. Para se encontrar o ponto de equilíbrio, aplicou-se o cá1culo diferencial na equação (3):

$$
\begin{aligned}
& \frac{\mathrm{dY}_{\mathrm{e}}}{\mathrm{dX}}=\mathrm{a}-2 \mathrm{bX} ; \text { mas } \mathrm{a}-2 \mathrm{bX}=0 \text {, } \\
& \text { então } x_{0}=a / 2 b \quad \ldots \ldots \ldots \ldots \ldots \text { (4). }
\end{aligned}
$$

Para se obter a produção max́ima sustentāvel téorica, usando-se o valor de esforço ótimo $\left(X_{0}\right)$ na equação (3), temos:

$$
\mathrm{Y}_{\max }=\left(\mathrm{a}-\mathrm{bX}_{0}\right) \mathrm{X}_{0} \ldots \ldots \ldots \text { (5). }
$$

Se colocarmos o custo de operação na equação (3), e calculando-se o valor da renda bruta $\left(\mathrm{R}_{\mathrm{e}}\right)$ no lugar da captura total $\left(\mathrm{Y}_{\mathrm{e}}\right)$, podemos obter a variação do capital investido e o lucro previsto.

Aplicando-se o preço de unidade do pescado ( $p$ ) na equação (3), temos :

$\mathrm{R}_{\mathrm{e}}=\mathrm{p}(\mathrm{a}-\mathrm{bX}) \mathrm{X}$

onde $p=\operatorname{Cr} \$ 10.000,00 /$ ton. (preço médio da sardinha no 1 o semestre de 1980 em Santa Catarina). A equação (6) é chamada de curva de rendimento economico,

$\mathrm{C}=\mathrm{cX}$

onde $C$ é o custo total de operação e c é o custo de operação por unidade de esforço de pesca. Neste caso, utilizamos o custo de operação por lance de pesca. 0 relatório do GPE-Sardinha (PDP/SUDEPE, 1980) apresentou o custo de operação, por viagem de pesca, no valor de $\operatorname{Cr} \$ 71.040,00$ em agosto de 1980 (Tab. II), nele estando incluídas todas as despesas de operação. 0 mesmo trabalho mostra que o numero médio de lances por viagem foi 1,2 lances.

Então, podemos obter o custo de operação por lance no valor de $\operatorname{Cr} \$ 59.200,00$. 0 lucro (L) poderá ser apresentada pela equação:

$L=R_{e}-C=p(a-b x) x-c X \ldots$.

que é chamada de curva de rentabilidade. Aplicando-se o cálculo diferencial na equação (8), podemos estimar o nível de esforço ótimo para a obtenção do lucro máximo:

$\frac{d L}{d X}=a p-c-2 b p X, \quad$ mas $a p-c-2 b p X=0$, 
$\therefore \mathrm{x}_{\mathrm{oe}}=(\mathrm{ap}-\mathrm{c}) / 2 \mathrm{~b} \mathrm{p}$. Entretanto, podemos calcular o lucro máximo com a seguinte equação:

$$
\mathrm{L}_{\max }=(a p-c)^{2} / 4 b p \quad \ldots \ldots \ldots \ldots \text { (9). }
$$

A equação (9) demonstra que o lucro máximo poderá ser apresentado em função de duas variáveis: custo de operação por unidade de esforço (c) e o preço de pescado (p).

\section{Revisão dos dados do esforço de pesca}

Para se aplicar o modelo descritivo de Schaefer, temos de calcular os valores de captura por unidade de esforço (CPUE). Para o cálculo da CPUE, a padronização do esforço de pesca é muito importante, Por exemplo, se a capacidade do esforço de pesca aumentar no decorrer do tempo, não poderemos calcular a CPUE simplesmente dividindo a captura total pelo esforço de pesca, pois o aumento da capacidade de captura traria uma distorção no resultado do cálculo da CPUE.

No caso da frota da sardinha, não foi feito o acompanhamento do aumento da capacidade dos petrechos de pesca. Então, para a padronização do esforço, calculamos o tamanho médio dos barcos baseados na tabela de seus registros (PDP/SUDEPE, 1980) (Fig. 1). Podemos observar que, durante o período de 1969 a 1974, houve um aumento considerável no tamanho dos barcos. Porém, a partir de 1975, a capacidade média dos barcos estabilizou-se ao níve1 de 59 toneladas.

Para se verificar o efeito do aumento no tamanho dos barcos sobre a eficiência de captura, plotamos, no mesmo gráfico, a variação de captura por lance de pesca. Podemos notar que a captura por lance de pesca aumentou gradativamente durante o período de 1964 a 1971 , acompanhando o aumento do tamanho médio dos barcos. Após esta data, a captura por lance estabilizou-se ao níve1 de 10 a 11 toneladas, com exceção de 1972 e 1976, quando ocorreu baixa produção de sardinha nos Estados de São Paulo e Santa Catarina. Nos ültimos anos (1978 e 1979), foi observado novamente um pequeno aumento de captura por lance.

Com esta figura, podemos concluir que, para a padronização do esforço de pesca, podemos utilizar o tamanho médio de barcos que, aparentemente, corresponde à capacidade de captura. Supondo que

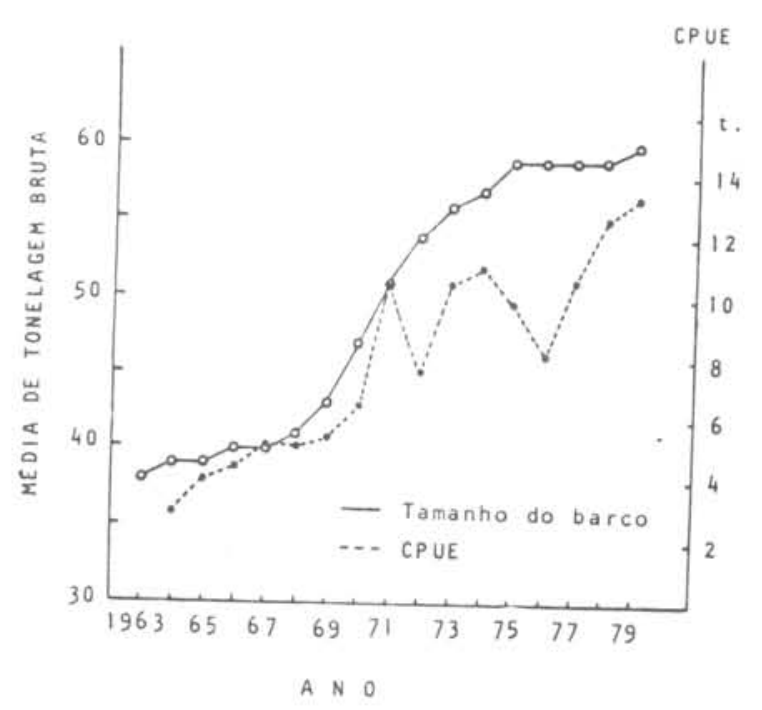

Fig. ! - Aumento do tamanho médio de barco de traineira, nos três Estados, e a variação de captura por lance de pesca (CPUE), observada nos Estados de São Paulo e Santa Catarina.

a capacidade de esforço de pesca em 1979 é igual a 1,00 , podemos corrigir o esforço de pesca nos anos anteriores, de acordo com o tamanho médio dos barcos.

Quanto aos dados estatísticos do esforço de pesca, os únicos disponíveis para os Estados do Rio de Janeiro, São Paulo e Santa Catarina, são o número total de viagens no período compreendido de 1969 a 1979 e o número total de lances de pesca para o período de 1974 a 1979. Usando estes dados, calculamos o esforço de pesca corrigido pelo tamanho médio dos barcos e a captura por unidade de esforço de pesca (lance e viagem)

(Tab. I).

\section{Resul tados}

\section{Curva de redimento}

Para a obtenção das constantes $a$ e $b$ da equação (1), calculamos a regressão linear pelo método de mínimo quadrado. 0 coeficiente de correlação entre CPUE e número de viagens foi baixo, tanto para o período de 1969 a 1979 ( $\mathrm{R}=$ $-0,15)$, quanto para o de 1974 a 1979 $(\mathrm{R}=-0,29) \dot{\sim}_{\text {. }}$ Entretanto, o coeficiente de correlação entre CPUE e número de lance foi alto $(R=-0,66)$. Aqui, utilizamos o nümero de lances como repiesentante do esforço de pesca para o cál- 
Bolm Inst. oceanogr., S Paulo, 30(1), 1981

Tabela I. Dados de captura e esforço de pesca da sardinha na região sudeste do Brasil

\begin{tabular}{|c|c|c|c|c|c|c|c|c|c|}
\hline Ano & $\begin{array}{c}\text { Captura } \\
\text { total }\end{array}$ & $\begin{array}{l}\text { Captura* } \\
\text { registrada }\end{array}$ & $\begin{array}{c}\text { Esforço de pesca } \\
\text { (No de lances) }\end{array}$ & $\begin{array}{l}\text { Esforço } \\
\text { corrigido }\end{array}$ & $\begin{array}{c}\text { CPUE } \\
\text { (Cap/lance) }\end{array}$ & $\begin{array}{l}\text { Esforço de pesca } \\
\text { (N: de viagens) }\end{array}$ & $\begin{array}{l}\text { Esforço } \\
\text { corrigido }\end{array}$ & $\begin{array}{c}\text { CPUE } \\
\text { (Cap/viagem) }\end{array}$ & $\begin{array}{l}\text { Taxa de } \\
\text { correçāo }\end{array}$ \\
\hline 1969 & 113.877 & 80.633 & - & - & - & 9870 & 7008 & 11,5 & 0,71 \\
\hline 1970 & 135.400 & 100.760 & - & - & - & 11473 & 8949 & 11,3 & 0,78 \\
\hline 1971 & 160.925 & 120.484 & - & - & - & 12208 & 10377 & 11,6 & 0,85 \\
\hline 1972 & 170.706 & 127.320 & - & - & - & 11824 & 10642 & 11,9 & 0,90 \\
\hline 1973 & 228.448 & 202.627 & - & - & - & 16005 & 14885 & 13,6 & 0,93 \\
\hline 1974 & 200.714 & 130.212 & 17886 & 17170 & 7,58 & 10591 & 10167 & 12.8 & 0,96 \\
\hline 1975 & 184.167 & 181.291 & 28437 & 27868 & 6,50 & 11748 & 11513 & 15,7 & 0,98 \\
\hline 1976 & 93.899 & 73.718 & 10615 & 10508 & 7,02 & 6268 & 6205 & 11,9 & 0,99 \\
\hline 1977 & 145.576 & 116.070 & 23067 & 22836 & 5,08 & 13161 & 13029 & 8,9 & 0.99 \\
\hline 1978 & 140.927 & 124.820 & 22269 & 22046 & 5,66 & 13626 & 13490 & 9,3 & 0.99 \\
\hline 1979 & 149.542 & 136.139 & 10645 & 10645 & 12,79 & 8653 & 8653 & 15.7 & 1.00 \\
\hline
\end{tabular}

- Captura registrada na ficha de bordo para cálculo de captura por unidade de esforço (CPUE).

culo da curva de rendimento. A relação entre CPUE e número de lances é apresentada na Figura 2, pela equação:

$$
\begin{aligned}
& \overline{\mathrm{U}}=12,26-0,000260 \mathrm{X} . \\
& \mathrm{Y}_{\mathrm{e}}=(12,26-0,000260 \mathrm{X}) \mathrm{X} \ldots
\end{aligned}
$$

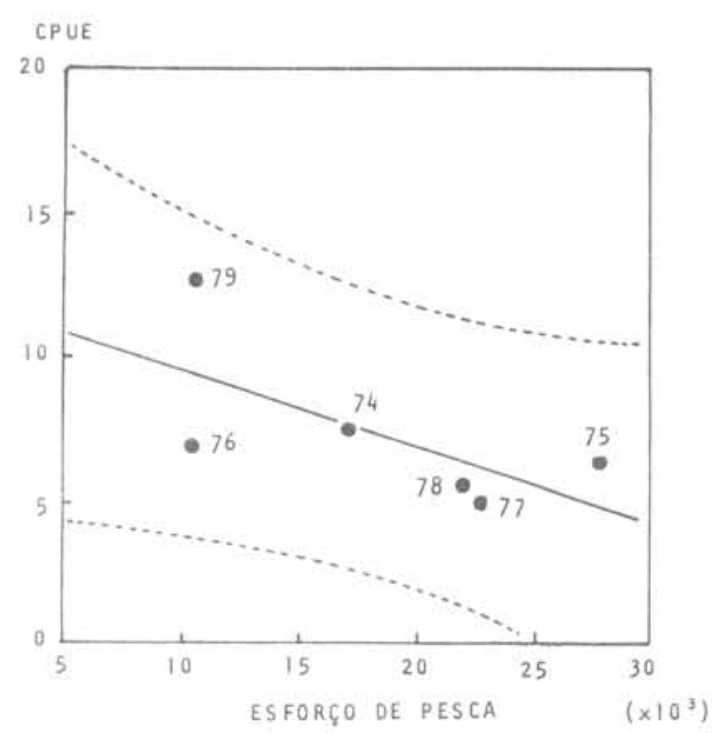

Fig. 2 - Correlação entre captura por unidade de esforço de pesca ( $n$ ? de lances) e o esforço de pesca observados nos três Estados (RJ, SP e SC).

A produção máxima sustentável no ponto de equilíbrio ( $\mathrm{P}_{\mathrm{e}}$ ) é de 144.527 toneladas por ano e o numero de lances correspondentes a esta produção é de 23.577 lances. Na Figura 3, é apresentada a curva de rendimento da equação (10) e as capturas registradas no mesmo período.

Curva de rendimento econômico e o cálculo de lucros

Os dados de captura e esforço de pesca registrados na ficha de bordo do PDP/ SUDEPE, não cobriram todos os barcos que estão operando nesta região. A média de captura registrada na ficha de bordo durante o período foi de, aproximadamente, $83,3 \%$ da captura total (Tab. I). Para o cálculo da renda bruta, utilizando-se a equação (6), as constantes $a$ e $b$ foram corrigidas para o valor total de captura, sendo obtida a seguinte equação:

$\mathrm{Y}_{\mathrm{e}}^{\prime}=\left(\mathrm{a}^{\prime}-\mathrm{b}^{\prime} \mathrm{X}\right) \mathrm{X}=$

$(12,26-0,00021667 \mathrm{X}) \mathrm{X}$

A partir desta equação, podemos calcular a produção máxima sustentável corrigida ( $\mathrm{Y}_{\max }=173.429$ ton./ano $)$ e o esforço de pesca correspondente a esta produção $\left(X_{0}=28.292\right.$ lances/ano $)$. Aplicando-se o preço do pescado

$(\mathrm{p}=\operatorname{Cr} \$ 10.000,00 /$ ton.) na equação (11), temos :

$\mathrm{R}_{\mathrm{e}}=\left(\mathrm{pa}^{\prime}-\mathrm{pb} \mathrm{x}\right) \mathrm{x}=$

$(122600-2,1667 \mathrm{x}) \times \ldots \ldots \ldots \ldots$ (12).

Aplicando-se o valor do esforço ótimo $\left(X_{0}\right)$ na equação (12), podemos obter a renda máxima teórica: 


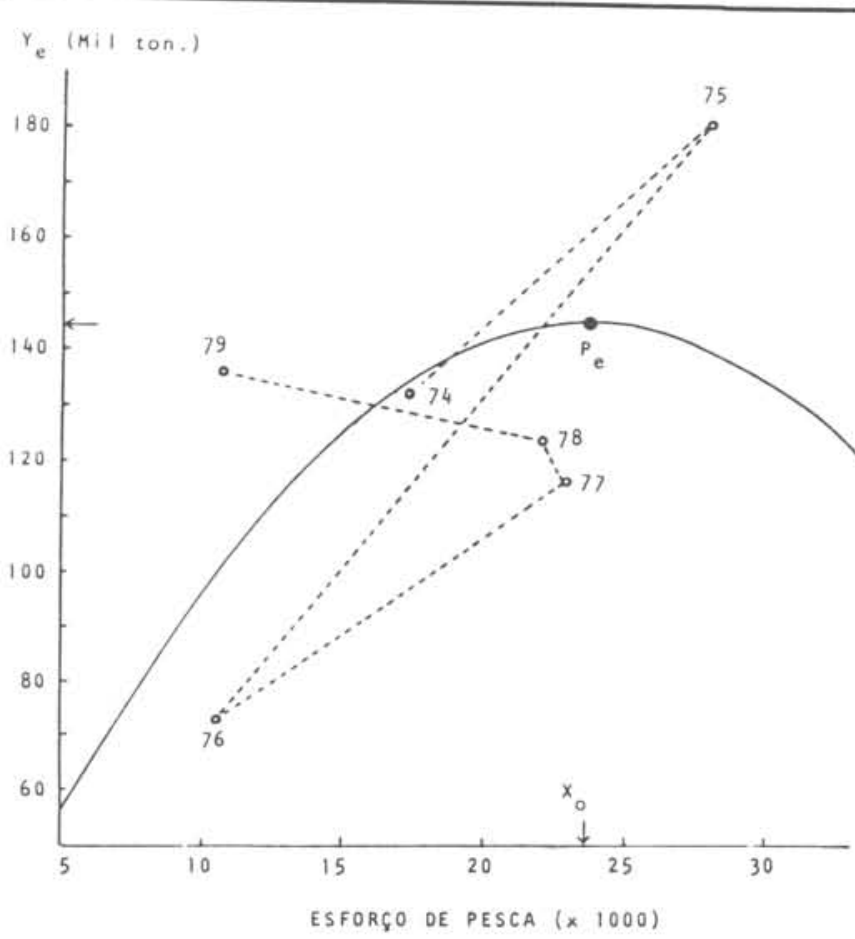

Fig. 3 - Curva de rendimento e o ponto de equilíbrio para obter a produção mäxima sustentāvel.

$$
\mathrm{R}_{\max }=\operatorname{Cr} \$ 1.734 \times 10^{6} .
$$

0 cálculo de lucro máximo foi feito da seguinte maneira:

$$
L=R_{e}-C=(63400-2,1667 X) X \ldots \text { (13). }
$$

0 nível do esforço ótimo de pesca, correspondente à produção do lucro máximo, seria:

$$
\begin{aligned}
& X_{o e}=\left(a^{\prime} p-c\right) / 2 b^{\prime} p=14.631 \text { 1ances. } \\
& \therefore \quad L_{\max }=\operatorname{Cr} \$ 464 \times 10^{6} .
\end{aligned}
$$

\section{Discussão}

A aplicação do modelo descritivo para a avaliação de estoque foi feita pela primeira vez no Brasil em 1973, pelo grupo de trabalho e treinamento (PDP/SUDEPE, 1974). Naquela época, havia pouca disponibilidade de dados sobre a captura e esforço de pesca da sardinha, principalmente no Estado do Rio de Janeiro, onde não existiam informações sobre o esforço de pesca. Então, para se calcular os valores da CPUE foram utilizados os dados do esforço de pesca observados nos Estados de São Paulo e Santa Catarina.
Nessa ocasião, não foi feita a padronização do esforço de pesca, devido à falta de dados sobre o aumento da eficiência de captura.

Os resultados da CPUE obtidos com o esforço de pesca-não-corrigido não apresentou nenhuma correlação com o aumento do esforço de pesca. Portanto, para se encontrar um índice de densidade média da população, utilizou-se o número de lances por dia no lugar da CPUE, o que não è real. E, dividindo-se a captura total por lance/dia, foi calculado o indice do esforço total hipotético. Todos os trabalhos de cálculo da produção máxima sustentáve1, elaborados posteriormente pelo Grupo Permanente de Estudos da Sardinha, foram feitos baseados neste procedimento. Porém, para o cálculo da curva de rendimento econômico, é necessário que tenhamos o valor real do esforço de pesca. Neste sentido, contando com a disponibilidade dos dados do Projeto Matriz de Pesca (PDP/SUDEPE, 1979) e com os do relatório apresentado na reunião anual do GPE-Sardinha (outubro de 1980), tentou-se calcular os valores da CPUE, com esforço de pesca em número de lances e de vagens. O ünico dado que apresentou alta taxa de correlação entre CPUE e esforço de pesca foi o número de lances, observado nos três Estados $(R=-0,66)$.

0 resultado da curva de rendimento calculado com os dados da ficha de bordo foi corrigido com a captura total na equação (11), sendo obtida a produção máxima sustentável da sardinha, no valor de 173.429 toneladas ao ano. Aplicandose o preço do pescado nessa equação, calculou-se a curva de rendimento econômico (Fig. 4), onde foi plotada a reta de custo de operação. Nessa figura, a linha AC significa a renda bruta ao nível de esforço no ponto Ce, a linha BC, o custo de operação. O lucro é representado pela linha $A B$. A área marcada com linhas diagonais significa a variação de lucros. Após o ponto de esforço ótimo $\left(\mathrm{x}_{\mathrm{o}}\right)$, onde o rendimento máximo é obtido, o aumento do esforço de pesca trará diminuição de rendimento e de lucro. No ponto D, o lucro é zero. A partir deste ponto, o aumento do esforço de pesca acarretará somente prejuízos.

Us ando-se a equação (13), foi calculada a variação do lucro, correspondente aos vārios nîveis de esforço de pesca. 


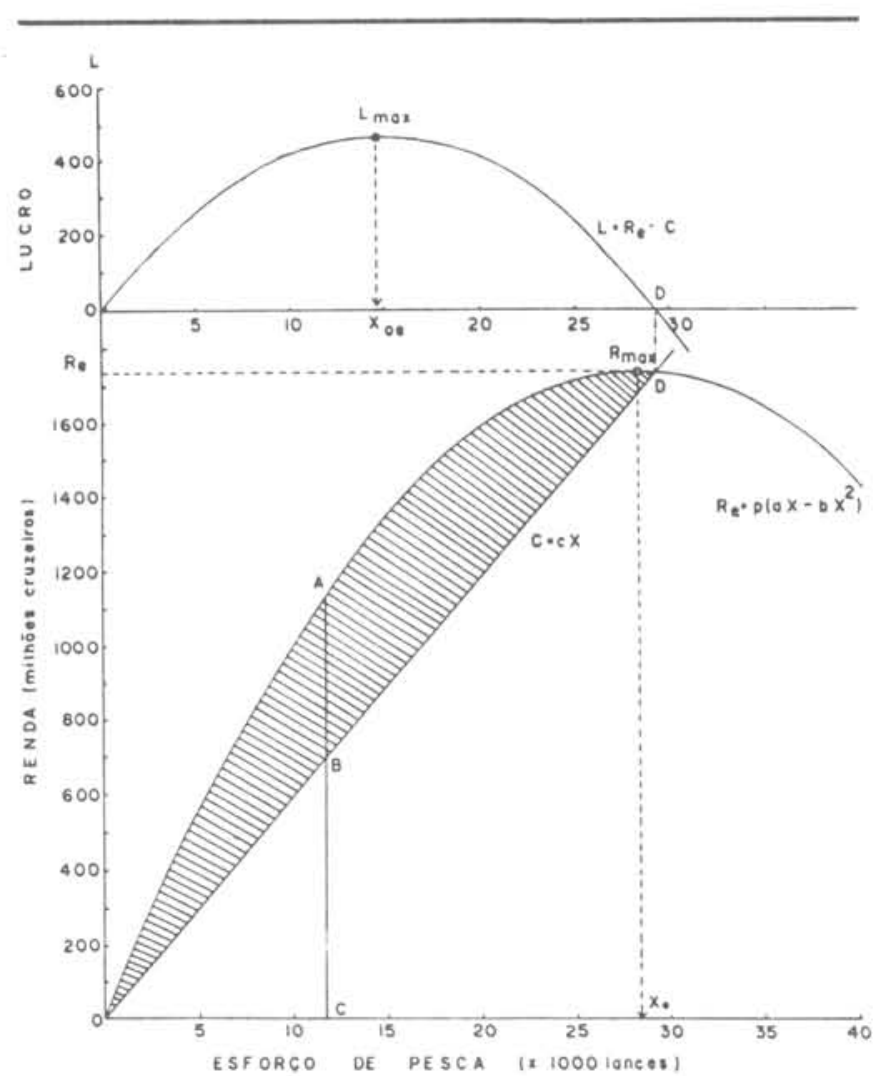

Fig. 4 - Curva de rendimento econômico $\left(R_{e}\right)$ e curva de rentabilidade (L).

A reta C apresenta o custo de operação.

$D=$ ponto de deficit, $R_{\max }=$ rendimento mäximo, $X_{0}=$ esforço ötimo para obter $R_{\max }, L_{\max }=$ lucro mäximo, $x_{o e}=$ esforço öt imo para obter $L_{\max }$.

0 nível de esforço de pesca, para obtenção do 1ucro máximo $\left(x_{o e}\right)$, é de 14.631 lances, inferior ao do esforço ótimo $(\mathrm{X})$. Do ponto de vista económico, o nível de esforço de pesca deve ser mantido no ponto $\mathrm{X}$; porém, se for o caso de se fornecer maior quantidade de proteínas animais para a população, o nível ideal corresponde ao ponto $x_{0}$.

A Figura 4 apresenta a curva de rendimento econômico, computada ao valor médio do pescado capturado no 10 semestre de 1980. Entretanto, variando o preço do pescado e o custo de operação, podemos obter outro tipo de comportamento na variação de lucros.

0 preço médio da sardinha apresentouse alto no 1 o trimestre de 1980 (Cr $\$ 12,55)$ e baixo no 2 ? trimestre $(\operatorname{Cr} \$ 8,76)$, no Estado de Santa Catarina. Em consequência do baixo preço da sardinha, os armadores e pescadores paralizaram suas atividades, para evitar prejuízo. Uma das medidas solicitadas pelos produtores ao Governo, foi o de subsidiar o custo de operação (combustível), desde que o preço do pescado, sendo determinado pela lei da oferta e demanda, impedia o Governo de interferir no preço de atacado. Porém, analisando-se o custo de operação na Tabela II, podemos notar que o custo do combustível ocupa somente uma pequena parcela (13\%) do custo total e que outros itens são indispensáveis, de modo que há pouca possibilidade de se

Tabela II. Custo de operação da pesca de sardinha no Estado de Santa Catarina (SC/PDP-DECOP).

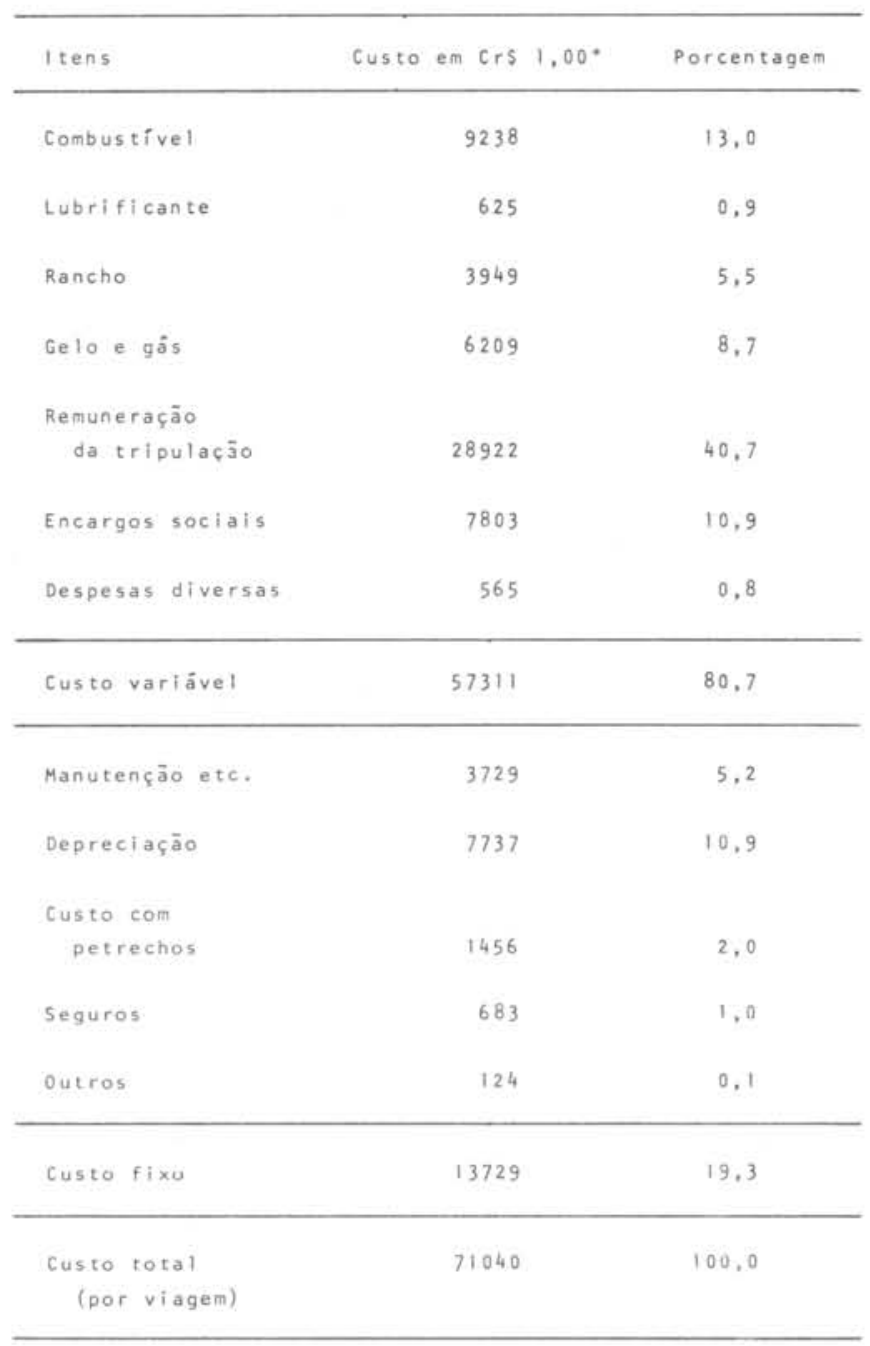

(*agosto de 1980)

diminuir este custo. Apesar deste fato, se conseguissemos reduzir o custo le operação em $20 \%$ do valor atual, a reta de 
custo mudaria de $\mathrm{C}-0$ para $\mathrm{C}-1$ na Figura 5. Consequentemente, aumentaria a ărea de lucro e o ponto de deficit deslocarse-ia de $\mathrm{D}_{0}$ para $\mathrm{D}_{1}$.

Em agosto e setembro de 1980 , o preço da sardinha nos Entrepostos de Santos e Itajaí baixou tanto, que chegou a menos de $\operatorname{Cr} \$ 6,00 / \mathrm{kg}$. A curva de rendimento econômico $\left(\mathrm{R}_{\mathrm{e}}-1\right)$ computada ao preço de $\operatorname{Cr} \$ 6,00 / \mathrm{kg}$ foi plotada na Figura 5. Neste caso, a margem de lucro quase desapareceu e o ponto de deficit deslocou-se de $\mathrm{D}_{0}$ para $\mathrm{D}_{2}$. Nesta situação, a única maneira de se evitar o prejuízo é paralizar a atividade de pesca, o que aconteceu. Este fato significa que, para se poder aumentar o nível de esforço de pesca ao nível de produção máxima sustentāvel (= renda máxima), o preço da sardinha tem de ser, pelo menos acima de Cr\$ $10,00 / \mathrm{kg}$.

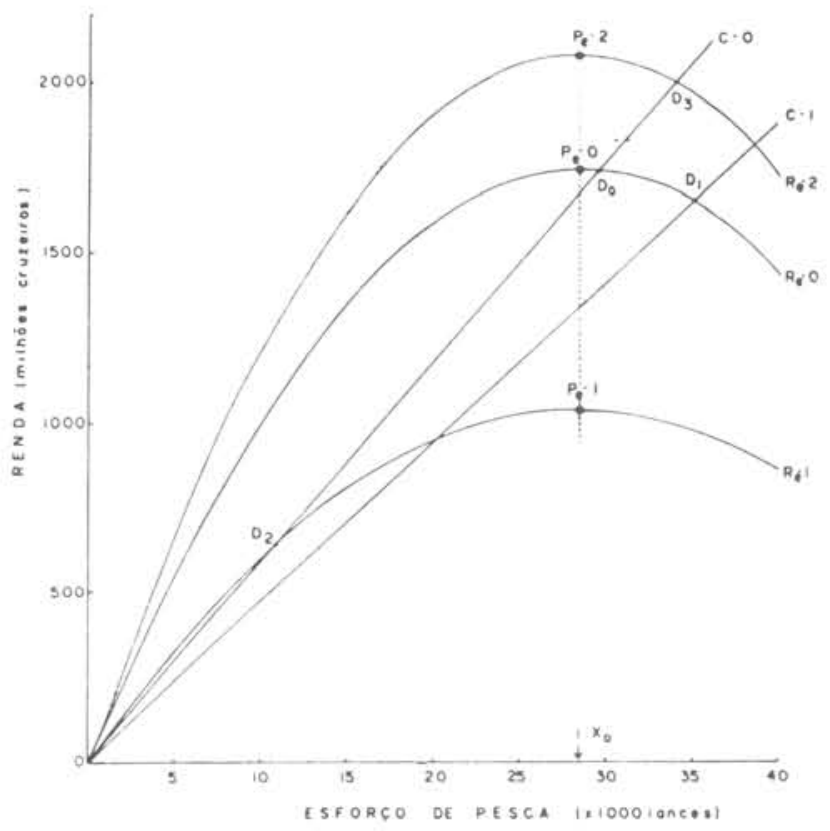

Fig. 5 - Curvas de rendimento econômico calculadas a 3 niveis de preço do pescado. $R_{e}-0 \quad(p=C r \$ 10,00 /$ kg), $R_{e}-1 \quad(p=C r \$ 6,00 / k g), R_{e}-2$ $(p=\operatorname{Cr} \$ 12,00 / \mathrm{kg}) . \quad \mathrm{C}-0=$ reta de custo de operação calculada com o valor de $\mathrm{Cr} \$ 59.200,00 /$ lance, $\mathrm{C}-1$ = reta de custo de operação calculada com o valor de $\operatorname{Cr} \$ 47.360,00 / 1$ ance.

Se conseguíssemos elevar o preço da sardinha ao valor de $\operatorname{Cr} \$ 12,00 / \mathrm{kg}$, a curva se deslocaria de $R_{e}-0$ para $R_{e}-2$,
- que daria maior margem de 1ucro. A Figura 6 apresenta a variação da curva de rentabilidade computada com o custo de operação fixo ( $\mathrm{Cr} \$ 71.040,00 /$ viagem ou Cr\$59.200,00/1ance). Podemos observar que o ponto de lucro máximo varia de acordo com o preço do pescado.

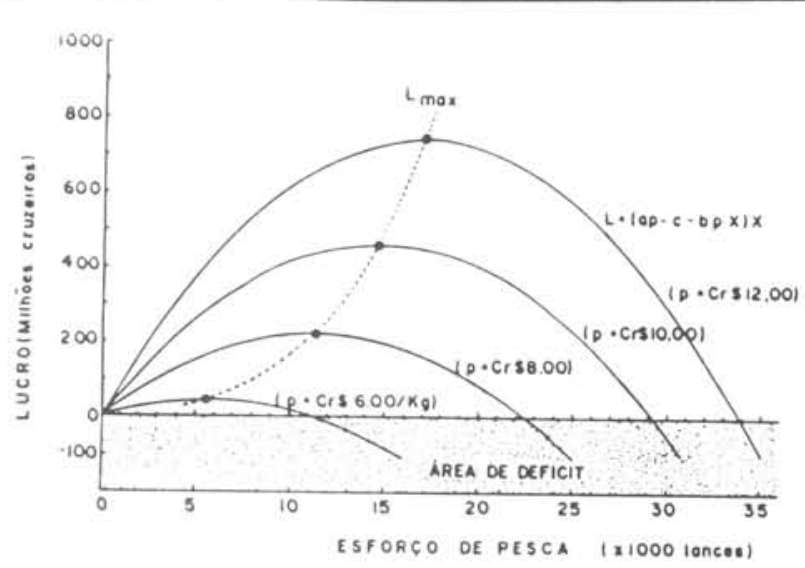

Fig. 6 - Curvas de rentabilidade computadas com diferentes preços do pes cado.

0 custo de operação apresentado na Tabela II é o mínimo necessário para a produção da sardinha. Portanto, para se aumentar a produção da sardinha e rentabilidade no setor, è necessārio aumentar o preço do pescado.

Considerando os resultados da anālise econômica da produção, podemos chegar às seguintes conclusôes:

1) A produção máxima sustentāvel, estimada através do modelo descritivo, é de aproximadamente $173 \mathrm{mil}$ toneladas ao ano nesta região. 0 esforço de pesca necessārio para esta produção é de 28.000 1ances ao ano;

2) Com o custo de operação por viagem no valor de Cr\$71.040,00, o preço da sardinha tem de ser acima de Cr $\$ 10,00 / \mathrm{kg}$ (preço do 1 9 desembarque);

3) Abaixo desse valor, a pesca da sardinha dará prejuízo ao setor de produção e não alcançará a produção mäxima sustentāvel.

Recomendações

A fim de se evitar crise na pesca da sardinha, podemos recomendar três medidas, com base nas conclusões acima:

1) Estabelecer o preço mínimo da s rdinha ao nível de $\operatorname{Cr} \$ 10,00 / \mathrm{kg}$ para o 
custo de operação atua1 (Cr\$71.040,00/ viagem). Conforme o aumento do custo, o preço mínimo deve ser ajustado;

2) 0 atual nível de esforço de pesca (300 barcos de traineira) é mais que suficiente para se capturar a produção máxima sustentāve1. Portanto, a atual Portaria no N-015 da SUDEPE, de 26 de agosto de 1977, que restringiu a entrada de novo esforço de pes$\mathrm{ca}$, deve ser mantida;

3) Considerando o fato de que existia grande defasagem entre o preço de entreposto e o de consumidor na ocasião da crise (i.e.e., Cr $\$ 6,00 / \mathrm{kg}$ no cais versus $\operatorname{Cr} \$ 60,00 / \mathrm{kg}$ na feira), - Governo deve procurar uma maneira de controlar a margem de lucro dos intermediārios.

Agradecimentos

Ao Prof. Dr. Plínio Soares Moreira do Instituto Oceanogräfico da Universidade de São Paulo, apresento meu especial agradecimento pela colaboração e revisão do trabalho.

E aos Srs. Geovanio Milton de Oliveira, Hiram Lopes Pereira, Ricardo de Deus Cardoso, Moaldo Fernando B. de Faria, Silvio Jablonski, do PDP/SUDEPE, e Sr. Helio Valentini do Instituto de Pesca de São Paulo, pelo fornecimento de valiosas informações.

Referências bibliogräficas

BEVERTON, R. J. H. \& HOLT, S. J. 1957. On the dynamics of exploited fish populations. Fish. Invest. Lond., ser. 2, 19:1-533.
PDP/SUDEPE. 1974. Relatörio da primeira reunião do grupo de trabalho e treinamento (GTT) sobre avaliação dos estoques. PDP/SUDEPE, sër. Doc. téc., (7) :1-149.

1.979. Relatörio do projeto Matriz da Pesca. Base de Operação do PDP.RJ .

1980. Relatório da reunião sardinhas. PDP/SUDEPE, Brasîlia.

RUSSEL, E. S. 1931. Some theoretical considerations on the "overfishing" problem. J. Cons. int. perm. Explor. Mer., 6:3-27.

SCHAEFER, M. B. 1954. Some aspects of the dynamics of populations important to the management of the commercial marine fisheries. Bull. inter-Am. trop. Tuna Commn, 1(2):27-56.

1957. A study of the dynamics of the fishery for yellowfin tuna in the eastern tropical Pacific Ocean. Bull. inter-Am. trop. Tuna Commn., 2(6):247-268.

SHINDO, S. \& YAGI, T. 1970. Study on the fisheries management in due consideration of biological and economical factors, with special reference to the Japanese pair traw1 fishery in the East China Sea and the Yellow Seas. Rep. Seikai reg. Fish. Res. Lab., (6):1-38.

(Recebido em 23/março/1981) 